\title{
Investigating key functions of hand movements by individuals with visual impairment: Improving teaching practices in special education through research
}

\author{
Vassilios Sotirios Argyropoulos*, University of Thessaly, Argonafton \& Filellinon St., 38221 Volos, Greece \\ Sofia Chamonikolaou, University of Thessaly, Argonafton \& Filellinon St., 38221 Volos, Greece
}

\section{Suggested Citation:}

Argyropoulos, V. S \& Chamonikolaou S. (2016). Investigating key functions of hand movements by individuals with visual impairment: Improving teaching practices in special education through research.

$$
\text { (1), 002-010. }
$$

Selection and peer review under responsibility of Assoc. Prof. Dr. Deniz Ozcan, Near East University, Cyprus. (C)2016 SciencePark Research, Organization \& Counseling. All rights reserved.

\begin{abstract}
Research is still ongoing with regard to types of exploratory movement by active touch and its key functions in individuals with visual impairment. The aim of the present study was to describe and identify different types of exploratory movement performed by individuals with visual impairment in their exploration of geometric shapes. A total of 12 participants were asked to explore a number of simple and complex geometric shapes. The research design consisted of two research phases. In the first phase, the participants were asked to describe and, if possible, to identify the properties of each shape. In the second phase, the participants were asked to describe their hand movements during active exploration. Finally, the research highlighted that by observing patterns of exploratory movement, educators of students with visual impairment can determine which strategies may be worth exploring with a view to their adoption in teaching practices and instruction.
\end{abstract}

Keywords: Visual impairment, active touch, geometric shapes, think-aloud protocols, laterality, teaching practices.

\footnotetext{
* ADDRESS FOR CORRESPONDENCE: Vassilios Sotirios Argyropoulos, University of Thessaly, Argonafton \& Filellinon St., 38221 Volos, Greece. 


\section{Introduction}

Tactile perception, as it is widely known, is a complex process based on information that an individual receives from an object or part of an object. Haptic perception, on the other hand, refers to the interpretation of a tactile stimulus, which leads to the formation of a perception (Piaget \& Inhelder, 1997). It is important to point out that even though the information comes from tactile exploratory movements, it is difficult to exclude information from other senses such as hearing during the above processes (Ballesteros \& Heller, 2008).

A number of research procedures can be applied to identify the properties of objects by active touch. Active exploratory movements refer to the actions taken by the individual to recognise an object. The main difference between active and passive exploratory movements is that the latter refer to the stimulus the individual receives from the object (Lederman \& Klatzky, 1987).

Several efforts have been made to classify the haptic procedures used in judging the geometric properties of objects. The type of exploratory movement used depends on the information an individual wants to extract from the object (Homa, Kahol, Tripathi, Bratton \& Panchanathan, 2009). Also, the information that an individual obtains through exploratory movements is associated with the object's global shape, exact shape, weight, volume, texture, material, temperature and function (Lederman \& Klatzky, 1987; Withagen, Kappers, Vervloed, Knoors \& Verhoeven, 2013).

Apart from exploratory movement types, handedness is also significant in respect to this investigation. Research on the lateralisation of hand function has given mixed results. Some studies report a left-hand advantage in recognizing objects, while others highlight advantages in using both hands and multiple fingers (Morash, Pensky, Tseng \& Miele, 2014). In fact, it has been observed that visual impairment affects not just the choice of hand but also the degree of handedness (Argyropoulos, Sideridis \& Papadimitriou, 2014). Finally, many studies of haptic object recognition do not mention or control handedness or permitted bimanual exploration (Craddock \& Lawson, 2009; Stone \& Gonzalez, 2014).

The aim of the present study was twofold: a. to describe and identify different types of exploratory movements performed by individuals with visual impairment during the exploration of geometric shapes (first research objective) and b. to describe the participants' hand preference during the exploration of geometric shapes (second research objective).

\section{Methodology}

\subsection{Participants}

The participants of the research study included twelve individuals ( age $=38.8$ years) with visual impairment who were recruited from two cities in Greece. Eight participants were congenitally blind and four were adventitiously blind with no additional disabilities (see Table 1).

Table 1. Characteristics of the blind participants

\begin{tabular}{lcll}
\hline \multicolumn{1}{c}{ Sex } & Age $^{\text {a }}$ & \multicolumn{1}{c}{ LV } & \multicolumn{1}{c}{ Cause } \\
\hline Congenitally blind & & & PHPV \\
F & 20 & FB & Optic nerve atrophy \\
M & 39 & FB & Optic nerve atrophy \\
F & 53 & FB & Congenital cataract \\
M & 56 & FB & RP \\
F & 49 & FB & RP \\
M & 46 & FB & RLF \\
M & 31 & 2 months &
\end{tabular}




\begin{tabular}{|c|c|c|c|}
\hline \multirow{2}{*}{\multicolumn{4}{|c|}{$\begin{array}{l}\text { M } \\
\text { Adventitiously blind }\end{array}$}} \\
\hline & & & \\
\hline$M$ & 41 & 16 years old & Glaucoma \\
\hline M & 50 & 16 years old & Glaucoma \\
\hline $\mathrm{F}$ & 49 & 16 years old & Iridocyclitis \\
\hline $\mathrm{F}$ & 42 & 16 years old & Glaucoma \\
\hline
\end{tabular}

\subsection{Material}

In the present study, we used the following six geometric shapes: square, circle, triangular prism, triangular pyramid, one complex 2D shape and one complex 3D shape (a complex shape is the combination of other basic geometric shapes). The shapes were constructed using different material such as wood, carton board and plastic. Also, they differed in texture, as some were covered with smooth carton board and velvet.

\subsection{Research design}

The research design comprised two research phases. In the first phase, the shapes were administered randomly to the participants, and, in turn, they were asked to comment on their properties, material and texture. In the second phase, the participants were requested to describe their hand movements when they explored the shapes (first research phase). Additionally, all participants were informed that the whole process would be video recorded, with the camera focusing only on their hands. The camera was positioned on a tripod behind each participant's right shoulder after the recording modes were set. Colour and sound were used in all recordings.

\subsection{Data analysis}

For the video analysis, the Avid Media Composer 7.0 program was used, which allows frame-byframe image resolution. The analysis of the exploratory movements was based on the protocol of movements developed by Lederman and Klatzky $(1987,1992)$. Finally, an analysis was conducted of the participants' think-aloud protocols obtained from both research phases. The data, regarding the properties of the shapes, were analysed and clustered into two thematic categories, namely, global properties and featural properties. Global properties refer to the properties which describe the shape as a whole (i.e., volume, overview by the outline, symmetry, etc.). In contrast, featural properties refer to properties associated with features of shapes such us texture, material, length of the sides of the geometric shapes and their geometrical characteristics.

\section{Results}

The results were grouped into two types of blindness: adventitious and congenital and into two types of properties: global and featural. Also, the researchers commented on results relevant on handedness and active exploration. It was decided to present the results according to the research phases as they were described above. 


\subsection{Results regarding types of hand movement by adventitiously and congenitally blind participants with respect to global properties of geometric shapes (first research objective)}

According to the results, the highest frequency of applied hand movements by the participants when they wanted to extract information about global properties were a. enclosure, $b$. contour following and c. lateral motion. This situation was noted in both research phases and the results are presented in Table 2 (data regarding the first research phase) and in Table 3, respectively, (data regarding the second research phase). In specific, the movement was applied by the majority of the participants who were adventitiously blind in both phases. In turn, the movement described as was applied by the participants to extract information regarding the outline of the shapes. The so-called seemed to help the participants who were adventitiously blind in extracting or confirming information on the shapes in question as a whole.

In contrast, the analysis of the congenitally blind participants in the first research phase showed that via , they fully grasped the global shape despite the fact that in each exploration they started their exploration with . Generally, it was observed that they conducted more movements when they began to describe the properties of the geometric shape, possibly in order to confirm the shape. The above finding was also confirmed during the second research phase when they were asked to describe the movements they conducted.

Table 2. Classification of exploratory movements regarding global properties of the shapes (first research phase)

\begin{tabular}{|c|c|c|c|c|c|c|c|c|}
\hline \multicolumn{9}{|c|}{ Exploratory movements } \\
\hline Shapes & $N_{\mathrm{E}}$ & $N_{\text {c. F. }}$ & N.M. & $N_{\text {P. }}$ & Ns. & Nu. H. & NH. о. & $N_{\text {c. M. }}$ \\
\hline \multicolumn{9}{|c|}{ Adventitiously blind } \\
\hline Sq. & 4 & 1 & 3 & 1 & 1 & 0 & 2 & 0 \\
\hline Circ. & 4 & 2 & 3 & 1 & 1 & 0 & 1 & 0 \\
\hline Tr. prism & 4 & 4 & 1 & 1 & 1 & 0 & 2 & 0 \\
\hline Tr. pyram. & 4 & 4 & 1 & 0 & 0 & 0 & 1 & 0 \\
\hline C. $2 \mathrm{D}$ & 3 & 4 & 1 & 4 & 2 & 0 & 2 & 1 \\
\hline C. 3D & 1 & 3 & 1 & 1 & 3 & 0 & 1 & 1 \\
\hline \multicolumn{9}{|c|}{ Congenitally blind } \\
\hline Sq. & 5 & 8 & 4 & 1 & 0 & 0 & 2 & 0 \\
\hline Circ. & 7 & 6 & 3 & 0 & 0 & 1 & 4 & 0 \\
\hline Tr. prism & 6 & 6 & 2 & 1 & 0 & 0 & 2 & 0 \\
\hline Tr. pyram. & 6 & 7 & 3 & 0 & 0 & 0 & 3 & 0 \\
\hline C. $2 \mathrm{D}$ & 5 & 7 & 6 & 3 & 1 & 0 & 0 & 0 \\
\hline C. 3D & 3 & 6 & 4 & 1 & 0 & 1 & 3 & 0 \\
\hline
\end{tabular}

$\mathrm{E}=$ number of participants' enclosures; $\quad$ C.F = number of participants' contour followings; L.M. = number of participants' lateral motions; P. = number of participants' pressures; $\mathrm{s}$. = number of participants' static holdings; $\quad$ и.н. $=$ number of participants' unsupporting holdings; н.о. = number of participants' hitting the object; c.M. number of participants' triangular prism; Tr. pyram. = triangular pyramid; $\mathrm{C} .2 \mathrm{D}=$ complex $2 \mathrm{D}$ shape; $\mathrm{C}$. 3D = complex 3D shape. 
Table 3. Classification of exploratory movements regarding global properties of the shapes (second research phase)

\begin{tabular}{|c|c|c|c|c|c|c|c|c|}
\hline \multicolumn{9}{|c|}{ Exploratory movements } \\
\hline Shapes & $N_{\mathrm{E}}$ & $N_{\text {c. F. }}$ & $N_{\text {L.M. }}$ & $N_{\text {p. }}$ & Ns. & Nu. н. & $N_{\text {H. о. }}$ & $N_{\text {c. M. }}$ \\
\hline \multicolumn{9}{|c|}{ Adventitiously blind } \\
\hline Sq. & 2 & 1 & 2 & 0 & 0 & 0 & 0 & 0 \\
\hline Circ. & 1 & 2 & 0 & 0 & 0 & 0 & 0 & 0 \\
\hline Tr. prism & 2 & 2 & 0 & 1 & 0 & 0 & 1 & 0 \\
\hline Tr. pyram. & 2 & 0 & 1 & 0 & 0 & 0 & 0 & 0 \\
\hline C. $2 \mathrm{D}$ & 4 & 3 & 1 & 0 & 0 & 0 & 0 & 0 \\
\hline C. 3D & 5 & 1 & 0 & 0 & 0 & 0 & 0 & 0 \\
\hline \multicolumn{9}{|c|}{ Congenitally blind } \\
\hline Sq. & 7 & 8 & 2 & 0 & 0 & 0 & 0 & 0 \\
\hline Circ. & 5 & 4 & 3 & 0 & 0 & 0 & 1 & 0 \\
\hline Tr. prism & 6 & 6 & 1 & 1 & 0 & 0 & 1 & 0 \\
\hline Tr. pyram. & 5 & 3 & 2 & 0 & 0 & 0 & 2 & 0 \\
\hline C. $2 \mathrm{D}$ & 6 & 5 & 5 & 1 & 0 & 0 & 1 & 0 \\
\hline C. 3D & 3 & 5 & 0 & 2 & 0 & 0 & 1 & 0 \\
\hline
\end{tabular}

$\mathrm{E}=$ number of participants' enclosures; $\mathrm{C.F}=$ number of participants' contour followings; L.M. = number of participants' lateral motions; $\quad$ P. = number of participants' pressures; s. = number of participants' static holdings; и.н. = number of participants' unsupporting holdings; H.O. = number of participants' hitting the object; c.M. number of

$;$ Sq. = square; Circ. $=$ circle; $\mathrm{Tr}$. prism $=$ triangular prism; Tr. pyram. $=$ triangular pyramid; $\mathrm{C} .2 \mathrm{D}=$ complex $2 \mathrm{D}$ shape; C. 3D = complex 3D shape.

It would be very interesting to look for significant differences between the two research phases but the small number of participants did not allow for such an analysis.

\subsection{Results regarding types of hand movement by adventitiously and congenitally blind participants with respect to featural properties of geometric shapes (first research objective)}

In both phases, the exploratory movements

and

were recorded as being used to extract information about featural properties. These movements also provided information to all the participants regarding the material and the length of the sides. Again, no statistical analysis was conducted between the two research phases because of the small number of participants.

Table 4. Classification of exploratory movements regarding featural properties of the shapes (first research phase)

\begin{tabular}{lcccccccc}
\hline \multicolumn{7}{c}{ Exploratory movements } \\
Shapes & $\boldsymbol{N}_{\text {E. }}$ & $\boldsymbol{N}_{\text {c. F. }}$ & $\boldsymbol{N}_{\text {L.M. }}$ & $\boldsymbol{N}_{\text {P. }}$ & $\boldsymbol{N}_{\text {s. }}$ & $\boldsymbol{N}_{\text {U. H. }}$ & $\boldsymbol{N}_{\text {H. O. }}$ & $\boldsymbol{N}_{\text {c. M. }}$ \\
\hline Adventitiously blind & & & & & & & & \\
Sq. & 1 & 2 & 2 & 1 & 0 & 0 & 1 & 1 \\
Circ. & 0 & 0 & 3 & 2 & 0 & 1 & 3 & 0 \\
Tr. prism & 2 & 3 & 4 & 3 & 1 & 0 & 3 & 4 \\
Tr. pyram. & 0 & 2 & 4 & 1 & 0 & 1 & 3 & 2 \\
C. 2D & 2 & 4 & 4 & 4 & 2 & 0 & 3 & 1
\end{tabular}




$\begin{array}{lllllllll}\text { C. } 3 \mathrm{D} & 1 & 4 & 4 & 2 & 2 & 0 & 1 & 3\end{array}$

Congenitally blind

$\begin{array}{lllllllll}\text { Sq. } & 0 & 4 & 6 & 3 & 0 & 0 & 5 & 1\end{array}$

$\begin{array}{lllllllll}\text { Circ. } & 0 & 2 & 7 & 4 & 0 & 1 & 4 & 1\end{array}$

$\begin{array}{lllllllll}\text { Tr. prism } & 1 & 4 & 7 & 7 & 0 & 0 & 4 & 5\end{array}$

$\begin{array}{lllllllll}\text { Tr. pyram. } & 1 & 6 & 6 & 1 & 0 & 0 & 4 & 4\end{array}$

$\begin{array}{lllllllll}\text { C. 2D } & 1 & 6 & 6 & 6 & 0 & 0 & 6 & 1\end{array}$

$\begin{array}{lllllllll}\text { C. 3D } & 4 & 5 & 7 & 4 & 1 & 0 & 5 & 3\end{array}$

$\mathrm{E}=$ number of participants' enclosures; $\mathrm{C.F}=$ number of participants' contour followings; L.M. = number of participants' lateral motions; $\mathrm{p}$. = number of participants' pressures; $\mathrm{s}$. = number of participants' static holdings; и.н. = number of participants' unsupporting holdings; H.O. = number of participants' hitting the object; c.m. number of participants' ; Sq. = square; Circ. $=$ circle; Tr. prism $=$

triangular prism; Tr. pyram. = triangular pyramid; C.2D = complex $2 \mathrm{D}$ shape;

C. $3 D=$ complex $3 D$ shape.

Table 5. Classification of exploratory movements regarding featural properties of the shapes (second research phase)

\begin{tabular}{|c|c|c|c|c|c|c|c|c|}
\hline \multicolumn{9}{|c|}{ Exploratory movements } \\
\hline Shapes & $N_{\mathrm{E}}$ & $N_{\text {c. F. }}$ & $N_{\text {L.M. }}$ & $N_{\text {P. }}$ & Ns. & Nu. н. & $\mathbf{N}_{\text {H. o. }}$ & Nc. M. \\
\hline \multicolumn{9}{|c|}{ Adventitiously blind } \\
\hline Sq. & 2 & 4 & 4 & 0 & 0 & 0 & 1 & 2 \\
\hline Circ. & 1 & 2 & 2 & 0 & 0 & 0 & 1 & 0 \\
\hline Tr. prism & 1 & 1 & 2 & 0 & 1 & 0 & 0 & 1 \\
\hline Tr. pyram. & 0 & 1 & 4 & 0 & 0 & 1 & 1 & 2 \\
\hline C. 2D & 1 & 3 & 3 & 3 & 0 & 0 & 1 & 1 \\
\hline C. 3D & 1 & 2 & 1 & 1 & 0 & 1 & 0 & 0 \\
\hline \multicolumn{9}{|c|}{ Congenitally blind } \\
\hline Sq. & 1 & 2 & 7 & 3 & 0 & 0 & 5 & 4 \\
\hline Circ. & 0 & 4 & 7 & 1 & 0 & 0 & 5 & 0 \\
\hline Tr. Prism & 2 & 6 & 8 & 0 & 0 & 0 & 4 & 4 \\
\hline Tr. pyram. & 2 & 7 & 8 & 2 & 1 & 0 & 6 & 6 \\
\hline C. 2D & 0 & 6 & 7 & 2 & 1 & 0 & 5 & 4 \\
\hline C. 3D & 4 & 4 & 7 & 2 & 0 & 0 & 7 & 4 \\
\hline
\end{tabular}

$\mathrm{E}=$ number of participants' enclosures; $C . \mathrm{F}=$ number of participants' contour followings; L.M. = number of participants' lateral motions; $\mathrm{p} .=$ number of participants' pressures; $\mathrm{s}$. = number of participants' static holdings; U.H. = number of participants' unsupporting holdings; H.O. = number of participants' hitting the object; c.M. number of participants' ; Sq. = square; Circ. = circle; $\mathrm{Tr}$. prism = triangular prism; Tr. pyram. = triangular pyramid; $C .2 \mathrm{D}=$ complex $2 \mathrm{D}$ shape; C. 3D = complex 3D shape. 


\subsection{Results regarding handedness in conjunction with exploratory movements applied by adventitiously and congenitally blind (second research objective).}

Finally, regarding hand preference (second research objective), it appeared from the video analysis that the left hand was used more for the exploratory movement , and the right was used more for the movement movements.

. Both hands were used for all other exploratory

Table 6. Exploratory movements and handedness by adventitiously and congenitally blind participants

\begin{tabular}{lcccccccccccccccccc}
\hline Shape & \multicolumn{3}{c}{ Square } & \multicolumn{4}{c}{ circle } & \multicolumn{4}{c}{ Tr. prism } & \multicolumn{3}{c}{ Tr. pyram } & \multicolumn{3}{c}{ C. 2D } & \multicolumn{3}{c}{ C. 3D } \\
& $\boldsymbol{N}_{\mathrm{L}}$ & $\boldsymbol{N}_{\mathrm{R}}$ & $\boldsymbol{N}_{\mathrm{B}}$ & $\boldsymbol{N}_{\mathrm{L}}$ & $\boldsymbol{N}_{\mathbf{R}}$ & $\boldsymbol{N}_{\mathrm{B}}$ & $\boldsymbol{N}_{\mathrm{L}}$ & $\boldsymbol{N}_{\mathrm{R}}$ & $\boldsymbol{N}_{\mathrm{B}}$ & $\boldsymbol{N}_{\mathrm{L}}$ & $\boldsymbol{N}_{\mathrm{R}}$ & $\boldsymbol{N}_{\mathrm{B}}$ & $\boldsymbol{N}_{\mathrm{L}}$ & $\boldsymbol{N}_{\mathrm{R}}$ & $\boldsymbol{N}_{\mathrm{B}}$ & $\boldsymbol{N}_{\mathrm{L}}$ & $\boldsymbol{N}_{\mathrm{R}}$ & $\boldsymbol{N}_{\mathrm{B}}$ \\
\hline E. & 9 & 3 & 0 & 3 & 5 & 3 & 2 & 4 & 0 & 8 & 2 & 2 & 5 & 3 & 4 & 7 & 1 & 4 \\
C. F. & 1 & 0 & 11 & 0 & 1 & 9 & 3 & 0 & 9 & 1 & 1 & 10 & 0 & 0 & 10 & 1 & 0 & 10 \\
L.M. & 0 & 0 & 9 & 2 & 3 & 7 & 3 & 0 & 8 & 1 & 1 & 9 & 2 & 0 & 8 & 1 & 1 & 9 \\
P. & 0 & 0 & 5 & 0 & 3 & 4 & 2 & 0 & 8 & 0 & 0 & 3 & 0 & 0 & 10 & 0 & 0 & 8 \\
S. & 0 & 0 & 1 & 0 & 1 & 0 & 0 & 0 & 1 & 0 & 0 & 0 & 0 & 0 & 3 & 1 & 0 & 3 \\
U. H. & 0 & 0 & 0 & 0 & 2 & 0 & 0 & 0 & 0 & 0 & 0 & 0 & 0 & 0 & 0 & 0 & 1 & 0 \\
H. O. & 0 & 5 & 4 & 0 & 2 & 0 & 1 & 5 & 2 & 3 & 2 & 3 & 2 & 6 & 2 & 0 & 3 & 3 \\
C. M. & 0 & 0 & 3 & 0 & 0 & 0 & 0 & 1 & 7 & 2 & 1 & 4 & 0 & 0 & 1 & 0 & 0 & 6 \\
\hline
\end{tabular}

$\mathrm{E}=$ number of participants' enclosures; $\quad$ C.F = number of participants' contour followings; L.M. = number of participants' lateral motions; $\quad \mathrm{p} .=$ number of participants' pressures; $\mathrm{s} .=$ number of participants' static holdings; U.H. = number of participants' unsupporting holdings; н.о. = number of participants' hitting the object; c M. number of participants'

Sq. = square; Circ. $=$ circle; Tr. prism $=$ triangular prism; Tr. pyram. $=$ triangular pyramid; $C .2 \mathrm{D}=$ complex $2 \mathrm{D}$ shape; $C$. 3D = complex 3D shape; $L=$ number of left hand use; $R=$ number of right hand use; $B=$ number of both hands use.

\section{Discussion}

The findings of the present study arise from the recording of exploratory movements and their relationship with the think-aloud protocols of the participants when they described and identified the properties of each shape, and when they described their hand movements during active exploration. It is suggested that with the movement , individuals with blindness receive information on the object's global properties, which is in line with the protocol of Lederman and Klatzky $(1987,1992)$. From the first research phase of the think-aloud protocol analysis, it can be seen that the participants who were congenitally blind described global properties using the movement, which may indicate that they needed to make more movements to confirm their judgment about the shape before describing it. This was also confirmed by the participants themselves in the second phase since they performed first and then proceeded to

In the case of featural properties, all participants emphasised that their choice regarding the type of their hand movement usually depends heavily on the information they wanted to perceive, for example, input regarding the material, the texture and/or the length of the sides of a shape. It is notable that the movement provided an optimum level of information on properties relating to texture and material $(1987,1992)$. The

exercise that was observed by the authors seemed to be employed more by the participants to determine the exact length of the sides of the geometric shapes.

The results for handedness showed that with , individuals with blindness tended to use the left hand, which is in agreement with other studies indicating that the left hand has an advantage in terms of object recognition (Craddock \& Lawson, 2009; Fagot, Hopkins \& Vauclair, 1993; Fagot, Lacreuse \& Vauclair, 1993, 1994) and in particular, recognition of the object's properties (Stone \& 
Gonzalez, 2014). The use of both hands for all other movements and the use of multiple fingers appeared to help the participants to identify and perceive the properties of the geometric shapes (Morash et al., 2014).

It is important to underline that the present study has some limitations. Due to the small sample, the participants' performance was not correlated with the two groups of the participants (congenitally and adventitiously blind). However, in a large-scale study, it would be interesting to assess their performance in relation to the inter-group correlation as well as handedness. Therefore, the follow-up of this study is the use of a handedness assessment tool. For instance, it is expected that the modified version of the Edinburgh Handedness Inventory and the Waterloo Handedness Questionnaire with relevant modifications for individuals with visual impairment and blindness (Argyropoulos et al., 2014) may work well for the needs of the present study.

In conclusion, the findings of the present study suggest that individuals with blindness employ certain exploratory strategies. Documenting the most effective strategies is essential for the education of individuals with visual impairment. Also, so far, it is unclear how individuals with visual impairment acquire exploratory strategies and whether these strategies vary according to the age of sight loss, previous visual experience, and tactile experience. Therefore, this research field is considered significant for the education of students with visual impairment. Apart from modifications associated with the environment and the material used, it is suggested that special teachers should take a number of important points into consideration when they want to include into their instructions types of haptic exploratory procedures. Those points may include: a) teaching students to use proper haptic exploratory strategies depending on the information they want to extract on each occasion (Homa et al., 2009; Lederman \& Klatzky, 1987, 1992; Withagen et al., 2013); b) prompting students to use both hands (Morash et al., 2014); c) prompting students to use all fingers and particularly index fingers (Morash et al., 2014); d) encouraging students to engage in training and rich tactile experiences to improve methods of manipulation (Warren, 1994); e) teaching exploratory procedures, not just using geometric shapes but also using real objects that students with visual impairment encounter in their day-to-day lives (Picard, Lebaz, Jouffrais \& Monnie, 2010) and f) gaining the appropriate training and knowledge to adopt tactile exploration strategies and teach students with visual impairment how to choose the right strategy for every occasion (Douglas, McCall, McLinden \& Pavey, 2009).

\section{References}

Argyropoulos, V., Sideridis, G. \& Papadimitriou, V. (2014). Hand preference of individuals with blindness in everyday activities: The effects of age of sight loss, age, and gender.

$$
\text { , 1885-1898. }
$$

Ballesteros, S. \& Heller, M. A. (2008). Haptic object identification. In M. Grunwald (Ed.),

Craddock, M. \& Lawson, R. (2009). Do left and right matter for haptic recognition of familiar objects? , 1355-1376.

Douglas, G., McCall, S., McLinden, M. \& Pavey, S. (2009).

Council for Special Education, Research Report, 3.

Fagot, J., Hopkins, W. D. \& Vauclair, J. (1993). Hand movements and hemispheric specialization in dichhaptic explorations. , 847-853.

Fagot, J., Lacreuse, A. \& Vauclair, J. (1993). Haptic discrimination of nonsense shapes: Hand exploratory strategies but not accuracy reveal laterality effects.

, 212-225. 
Fagot, J., Lacreuse, A. \& Vauclair, J. (1994). Hand-movement profiles in a tactual-tactual matching task: Effects of spatial factors and laterality.

, 347-355.

Homa, D., Kahol, K., Tripathi, P., Bratton, L. \& Panchanathan, S. (2009). Haptic concepts in the blind.

, 71(4), 690-698.

Lederman, S. J. \& Klatzky, R. L. (1987). Hand movements: A window into haptic object recognition. , 342-368.

Lederman, S. J. \& Klatzky, R. L. (1992). Stages of manual exploration in haptic object identification.

(6), 661-670.

Lederman, S. J. \& Klatzky, R. L. (2009). Haptic perception: A tutorial.

(7), 1439-1459.

Morash, V. S., Connell Pensky, A. E., Tseng, S. T. W. \& Miele, J. A. (2014). Effects of using multiple hands and fingers on haptic performance in individuals who are blind.

43, 569-588.

Piaget, J. \& Inhelder, B. (1997).

. London, UK: Routledge.

Picard, D., Lebaz, S., Jouffrais, C. \& Monnie, C. (2010). Haptic recognition of two-dimensional raised-line patterns by early-blind, late-blind, and blindfolded sighted adults.

224-235.

Stone, K. D. \& Gonzalez, C. L. R. (2014). Grasping without sight: Insights from the congenitally blind.

(10),e110175. doi:10.1371/journal.pone.0110175

Warren, D. H. (1994).

. Cambridge, UK: Cambridge University Press.

Withagen, A., Kappers, A. M. L., Vervloed, M. P. J., Knoors, H. \& Verhoeven, L. (2013). The use of exploratory procedures by blind and sighted adults and children. 1451-1464. 